\title{
Monte Carlo Analysis of Impact of Underascertainment of Mesothelioma Cases on Underestimation of Risk
}

\author{
Leonid Kopylev $^{*}, 1$, Patricia A. Sullivan ${ }^{2}$, Lisa C. Vinikoor ${ }^{1}$ and Thomas F. Bateson ${ }^{1}$
}

\author{
${ }^{I}$ National Center for Environmental Assessment, Office of Research and Development, US Environmental Protection \\ Agency, Washington, DC, USA \\ ${ }^{2}$ Field Studies Branch, Division of Respiratory Disease Studies, National Institute for Occupational Safety and Health, \\ Morgantown, WV, USA
}

\begin{abstract}
The accuracy of cancer mortality data varies across different cancers. Mortality records and death certificates may not always reflect the true cause of death for various reasons (e.g., misdiagnosis, improper recording on the death certificate, miscoding of the cause of death recorded on the death certificate). Mesothelioma, a rare tumor which is caused by exposure to asbestos, is a special case. Until 1999 when the $10^{\text {th }}$ revision of the International Classification of Diseases (ICD-10) introduced a specific mesothelioma code, mesothelioma deaths were coded to other causes (e.g., cancer of the pleura, cancer of other or ill-specified sites). Even after the introduction of this mesothelioma code, researchers have shown that estimates of mesothelioma mortality based on death certificates are still biased downward. This article reviews available literature with quantitative information on mesothelioma underascertainment, in particular on different rates of underestimation for pleural and peritoneal mesotheliomas, and suggests two approaches to estimating downward bias in absolute risk estimates due to mesothelioma underascertainment. The choice of approach used depends on whether the information on the proportion of peritoneal mesotheliomas is available. Both approaches are demonstrated and evaluated using a cohort of asbestos workers from Libby, MT. The methods developed in this article may be used in analyses of other asbestos cohorts and in methodologies to predict future mesothelioma burden in populations. Similar approaches can be used to assess the impact of underascertainment of other cancers on risk estimates of other chemicals.
\end{abstract}

Keywords: Pleural mesothelioma, peritoneal mesothelioma, Libby worker cohort, asbestos, death certificates, ICD codes.

\section{INTRODUCTION}

The literature on inaccuracies in cancer death certificates is extensive ${ }^{1}$. A seminal paper [2] on a large national (U.S.) study of hospital diagnoses for 48,836 cases of single primary cancers reported that the underlying cause of death was inaccurate in $35 \%$ of cancer deaths. When cancer mortality data for a specific cancer site are used, sufficient attention has to be paid to limitations of death certificate data. For a rare cancer like mesothelioma, attention to the source of the data and its limitations is paramount.

Mesothelioma is a rare tumor that arises from mesothelial cells in pleura, peritoneum, and, rarely, pericardium. The median survival from the time of diagnosis is less than one year and the upper $95^{\text {th }}$ confidence level on the survival time of mesothelioma patients in clinical trials rarely exceeds 2 years [3]. There is no association between mesothelioma and

\footnotetext{
*Address correspondence to this author at the National Center for Environmental Assessment, Office of Research and Development, US Environmental Protection Agency, 1200 Pennsylvania Ave NW MC 8623P, Washington, DC 20460, USA; Tel: 703-347-8599;

E-mail: kopylev.leonid@epa.gov
}

\footnotetext{
1 This article discusses results on cause of death accuracy based on publication from the USA and the UK. There is sufficient literature from many other countries, but “... significant differences exist among countries in the application of the rules for selecting the underlying cause of death differences that seriously affect cancer mortality statistics" [1].
}

smoking $[4,5]$. Exposure to asbestos is considered the most important and by far the most common cause of mesothelioma. Erionite, a naturally occurring fibrous mineral, is another cause [6], with possibly a strong genetic component [7]. The evidence for etiologic agents other than asbestos remains unclear. While there is some controversy [8] about mesothelioma and SV40 (Simian vacuolating virus 40), there is evidence suggesting [9] that asbestos and SV40 may act as co-carcinogens in the causation of mesothelioma. Therapeutic [10] and diagnostic (Thorotrast) [11-13] radiation have also been suggested as a cause of mesothelioma.

Mesothelioma was validated pathologically as a distinct diagnosis in 1931, and physicians and pathologists began to recognize mesothelioma as a primary tumor in the early 1960s [14]. In the London asbestos worker cohort [15], only 1 out of 6 pleural mesotheliomas and 3 out of 13 peritoneal mesotheliomas were mentioned on death certificates. The remaining pleural mesotheliomas were designated as lung cancers on death certificates, and the peritoneal mesotheliomas were listed as carcinoma or cancer of the gastro-intestinal tract. Several studies [16-19] were undertaken to investigate how well mesothelioma was captured by the International Statistical Classification of Diseases (ICD) codes and to identify what additional codes were reported when mesothelioma was listed on the death certificate or discovered during histopathological analysis. These studies are discussed in more detail below. 
While this article illustrates the use of Monte Carlo simulations to quantitatively address mesothelioma underascertainment, using the Libby amphibole asbestos worker cohort as demonstration, the methodology presented is not specific to this cohort.

This paper is organized as follows: Section 1 discusses the literature on mesothelioma underascertainment; Section 2 provides information about the Libby occupational cohort, mesothelioma ascertainment in that cohort, and statistical methodology; Section 3 proposes two approaches to quantitative estimation of the magnitude of underascertainment of mesothelioma; and the Discussion section presents the strengths and limitations of each approach.

\section{Underascertainment of Mesothelioma in the Asbestos Literature}

There are two ways to assess underascertainment: 1) one can follow mesothelioma patients with diagnosis documented by cancer registries and then compare their stated causes of death against their established diagnosis $[16,18,19]$ (this can be done for deaths before and after the establishment of a specific mesothelioma code for mesothelioma in ICD-10 [20] (Beginning in 1999, mesothelioma mortality was identified by ICD-10 code ' $\mathrm{C} 45$ '. Prior to that, there was no specific ICD code for mesothelioma); or 2) one can conduct histopathological analysis of all deaths in a cohort and compare those diagnostic causes of death to those reported on the death certificates $[17,21]$.

Comparing information from the cancer registries in Scotland to data from death certificates, Camidge et al. [19] sought to determine the ICD codes most frequently used for mesothelioma cases. The most frequently used ICD-9 [22] codes for individuals with mesothelioma were 163 (malignant neoplasm of pleura), followed by 162 (malignant neoplasm of trachea, bronchus and lung) and 199 (malignant neoplasm without specification of site). Also, of 607 mesotheliomas coded to ICD-10 in the Scotland cancer registry from 2000 to 2003 , only $75 \%$ were coded as mesothelioma [19]. These results for ICD-10 are somewhat lower than results below for ICD-9.

Pinheiro et al. [18] used Surveillance Epidemiology and End Results (SEER) data from nine geographic areas to evaluate differences between reported incidence (1998-1999 data) and mortality from mesothelioma as reported on death certificates (1999-2000 data), finding that 82\% of SEER incident mesothelioma cases were identified on death certificates after introduction of the unique ICD-10 code for mesothelioma 'C45.'

Linking Massachusetts Cancer Registry histopathologically confirmed mesothelioma incident cases 1982-1987 with 299 Massachusetts deaths through 1989, Davis et al. [16] reported that the ICD-9 codes used most frequently on death certificates were 199.0 and 199.1 (malignant neoplasm without specification of site, disseminated and other), 162.9 (malignant neoplasm of trachea, bronchus and lung, unspecified), and 163.9 (malignant neoplasm of pleura, unspecified). Only 12\% of mesothelioma incident cases were coded appropriately on death certificates according to ICD-9 coding rubrics for coding mesothelioma: 158.8 (malignant neoplasm of peritoneum, specified), 158.9 (malignant neoplasm of peritoneum unspecified) and 163 (malignant neoplasm of pleura). However, with manual inspection of death certificates, $83 \%$ of the individuals known to have mesothelioma could be identified (that is mesothelioma was recorded in some field of the death certificate). This study provides information on the likelihood that individuals who have been diagnosed as having mesothelioma will have that disease recorded (in some field) on their death certificate.

These reports $[16,18,19]$ used incidence data as the baseline for comparison.

Ribak et al. [17] used histopathological analysis to identify true mesothelioma cases from a large cohort of asbestos insulation workers $(17,800$ workers, 4,951 deaths, 301,593 person-years of follow-up, update by Selikoff and Seidman [21] of the cohort described by Selikoff et al. [23]). Overall, from 458 pathologically confirmed mesotheliomas, only $287 \quad(62.7 \%)$ death certificates mentioned mesothelioma. For pleural mesothelioma, $77.5 \%$ (134 of 173) of true cases were coded or mentioned on death certificates as any semantic form of the word "mesothelioma". Only 53.7\% of peritoneal mesotheliomas were noted on death certificates (153 of 285).

Selikoff and Seidman [21] extended the investigation [17] to specify misdiagnoses of mesothelioma as listed on the death certificate. Among 39 confirmed pleural mesotheliomas, the most common incorrect causes of death listed on the death certificates of confirmed mesothelioma cases were lung cancer $(n=23)$, other cancers $(n=7: 2$ cancers of the pleura, and single cases of stomach cancer, malignant histocytoma, thoracic neoplasm, colon cancer, and metastatic cancer), asbestosis $(\mathrm{n}=2)$, and other nonmalignant diagnoses $(n=7)$. Among 132 confirmed peritoneal mesotheliomas, the most common incorrect causes of death listed on the death certificates were abdominal cancer $(n=54)$, pancreatic cancer $(n=29)$ and cancer of the colon $(n=12)$. Many remaining misdiagnoses were in single digits. Among 311 mesotheliomas listed on death certificates and evaluated histopathologically, $24(7.7 \%)$ were other cancers, of which most were lung cancers $(n=21)$ [21]. This study provides information on the likelihood that individuals for whom medical records and pathology samples would support a diagnosis of mesothelioma will have mesothelioma recorded (in some field) on their death certificate.

Studies that confirmed the diagnosis of mesothelioma via cancer registries found $17-25 \%$ were not recorded on the death certificate while studies which confirmed mesothelioma by histopathological analysis of all deaths generally found $37 \%$ were missed. Peritoneal mesothelioma was missed more than pleural mesothelioma. Pleural mesothelioma was more often attributed as lung cancer while peritoneal was most often attributed as abdominal cancer. Recognizing this systematic underascertainment, knowledge of the frequency with which mesothelioma is underascertained can be applied to other cohorts of asbestosexposed people for whom only death certificates are available to estimate the true number of mesothelioma cases that might have actually occurred. 


\section{Libby Worker Cohort}

\section{2a. Cohort Description}

The term Libby amphibole refers to the unique fibrous amphibole asbestos which is found in the rocks and ore of Zonolite Mountain, 6 miles northeast of Libby, Montana. Zonolite Mountain contains a large vermiculite deposit which was mined from the early 1920s until 1990, primarily for use as loose-fill attic insulation. Vermiculite miners, mill workers and those in the processing plants were exposed to these amphibole fibers. Mortality and morbidity studies on the mine and mill workers from Libby have reported mesothelioma and lung cancer as well as other adverse health effects in these workers including pleural anomalies and interstitial fibrosis [24-31]. A recent paper [32] described the occurrence of mesothelioma among two Libby workers' family cases and nine residents in Libby, who had not worked in the mining or milling operations.

The NIOSH Libby worker cohort was used as an example to demonstrate how the Monte Carlo analysis described here can be used to estimate the number of cases of mesothelioma that may have been missed on death certificates. This cohort has been the focus of two epidemiologic investigations by NIOSH scientists resulting in five published reports [26-29,31]. Demographic and work history data were abstracted from company personnel and pay records. A database created by NIOSH in the 1980 s contained demographic data, work history, and vital status at the end of 1981 for 1,881 workers. A recent vital status update [31] compared these data with company records on microfilm, and work history data were re-abstracted to ensure data quality. One person was removed from the cohort because company records stated that he was hired but never worked. Nine workers with Social Security numbers listed in company records were excluded because demographic and work history data were not available. The final database included 1,871 study subjects.

Vital status follow-up was completed through 2006 using the National Death Index (NDI-Plus). Workers known to be alive [27] on or after January 1, 1979 (the date NDI began tracking deaths nationwide), but not found in the NDI search, were assumed to be alive on December 31, 2006. Nearly $54 \%$ of workers $(n=1,009)$ had died by December $31,2006$.

Exposure measurements available for the Libby worker cohort describe total fiber exposure, including both asbestiform and nonasbestiform fibers. Although exposure estimates beginning in the late 1960s includes data obtained using optical phase contrast microscopy (PCM) fiber analysis, earlier exposure estimates are based on total dust measurements in the workplace where sampling results from a midget impinger would be reported as million particles per cubic foot of air (mppcf). However, not all of those particles were fibers, or PCM visible fibers. Dust from the crushed rock would make up some portion of the total dust, including crystalline silica. A conversion factor to describe the relationship between total dust and fiber count was developed by Amandus and Wheeler [27].

\section{2b. Mesothelioma Ascertainment in the Libby Worker Cohort}

Beginning in 1999, mesothelioma mortality was identified by ICD-10 code ' $\mathrm{C} 45$ ' in the NDI-Plus records. Prior to adaption of revision 10 (1999), there was no specific ICD code for mesothelioma, but the rubrics of the various ICD revisions specified how to code mesothelioma deaths over different periods of time. NIOSH researchers obtained death certificates from the states. All death certificates were obtained for deaths prior to 1979 and coded to the ICD revision in effect at the time of death by a single National Center for Health Statistics-trained nosologist. After 1979, ICD codes were obtained from the NDI-Plus. For deaths occurring 1979-1998, death certificates were obtained if the NDI identified the death as being from one of the possible mesothelioma codes [33], or from respiratory cancer, nonmalignant respiratory disease, digestive cancer, or cancer unspecified. Death certificates (1940-1998) were reviewed by the NIOSH principal investigator [31] to identify any mention of mesothelioma on the death certificate. In total, 18 mesothelioma deaths occurring 1980-2006 were identified using these methods. The location of mesothelioma was known for only 6 of 18 mesothelioma deaths: 1 peritoneal and 5 pleural mesotheliomas were identified on death certificates.

\section{2c. Statistical Methodology}

The incidence of mesothelioma is extremely rare and the background rate in the general population without exposure is considered negligible [34]. Since there is a very low background risk, the exposure-response model applied here examines the relationship of the absolute risk of mesothelioma to asbestos exposure (as opposed to the relative risk model commonly applied for many other cancers, e.g. lung cancer). A Poisson model is employed here for estimating the absolute risk of mesothelioma, as the Poisson distribution is an appropriate model for use with data that are counts of a relatively rare outcome, such as observed mesothelioma deaths in the Libby worker cohort.

Estimation of the exposure-response relationship for mesothelioma using the Poisson model was performed using a Bayesian approach with a diffuse prior (WinBugs Version 1.4 [35]). The Poisson model has the following general form: $\mathrm{P}($ death $) \sim$ Poisson $(\mathrm{PY} * \lambda)$, where $\mathrm{PY}$ stands for personyears at risk and $\lambda$ is a product of a vector of regression coefficients $(\beta$ 's) and a vector of covariates. The benefit of using WinBugs is its computational ease and that it provides a posterior distribution of $\beta$ rather than just a point estimate. A diffuse (high variance) Gaussian distribution, truncated to exclude negative parameter values, is used as a prior. Use of diffuse priors is a standard procedure in Bayesian analysis when there is no prior knowledge under a particular model Standard practices of Monte Carlo Markov Chain (MCMC) analysis were followed for verifying convergence and sensitivity to the choice of initial values. The posterior distribution is based on 3 chains with a burn-in of 10,000 (i.e., the first 10,000 simulations are dropped) and thinning rate of 10 (i.e., only each $10^{\text {th }}$ simulation is used - thus 
reducing autocorrelation) such that 3000 total simulations constitute the posterior distribution of $\beta$. The mean of the posterior distribution served as a central estimate, the lower $5^{\text {th }}$ percentile served as a lower bound estimate, and the upper $95^{\text {th }}$ percentile of the distribution as an upper bound.

Compared with other exposure metrics available for the analysis (lagged and unlagged cumulative exposure, lagged and unlagged time-weighted exposure), duration of exposure provided the best fit for the cohort of 1871 Libby asbestos workers employed from 1935 to 1982, as measured by DIC (Deviance Information Criterion) and duration of exposure was used in the demonstration of Monte-Carlo analysis below. This is perhaps because duration of exposure is measured practically without error, while measurement of concentration of exposure is subject to the sizable measurement error (Section 2a), especially for the early years of operation, degrading the quality of fit.

\section{Monte Carlo Approaches to Quantifying Underesti- mation of Mesothelioma}

First, based on ICD codes identified in Davis et al. [16] and information from Selikoff and Seidman [21] and Marsh et al. [33], we identified the following pre-ICD-10 codes/revisions that mesothelioma could have been coded to (Table 1).

The ICD codes/revisions listed in Table $\mathbf{1}$ that are also present in the Libby worker cohort and pre-ICD-10 codes/revisions of confirmed mesothelioma cases are listed in Table 2.

Since type of mesothelioma (peritoneal or pleural) is only known for 6 of the 18 mesothelioma cases in the Libby worker cohort, we considered two approaches to estimating the underascertainment.

The first methodology is suitable when information on the proportion of peritoneal mesotheliomas is unknown. For this approach we simulated missed cases from a set of potential mesothelioma cases using a very wide definition of mesothelioma derived from Tables $\mathbf{1}$ and $\mathbf{2}$; this definition was thought to be broad enough to capture almost all true cases of mesothelioma. Not all of the deaths with ICD-codes corresponding to the wide definition of mesothelioma would be misdiagnoses of mesothelioma, so we used the proportion of missed mesotheliomas from Davis et al. [16], since the methods of ascertainment of mesothelioma cases in the Libby worker cohort is similar to the methods of Davis et al. [16]. The knowledge of the proportion of peritoneal mesotheliomas in the Libby worker cohort was disregarded.
The second methodology is suitable when the proportion of peritoneal mesotheliomas can be estimated or is known. For this approach we simulated missed cases from specific misdiagnoses, specific to pleural and peritoneal mesotheliomas [21]. Again, not all of the deaths with these diagnoses are misdiagnoses of mesothelioma and appropriate proportions were estimated from Selikoff and Seidman [21]. For this approach, we extrapolated the proportion of peritoneal mesothelioma cases from the 6 known cases to the 18 cases in the cohort.

Not enough information is yet available about ICD-10 codes misdiagnoses to include these in the tables. However, since published rates $[18,19]$ of misdiagnoses of mesothelioma deaths on the death certificates coded in ICD10 are no less than published rates [16] of misdiagnoses on death certificates coded in ICD-9, in both approaches, rates of misdiagnoses and specific misdiagnoses for ICD-10 mesothelioma cases were assumed to be similar to those of pre-ICD-10 mesothelioma cases.

\section{3a. Approach Using Wide Definition of Mesothelioma}

Using the misdiagnosis rate $17 \%$, estimated in Davis et al. [16], it was determined that approximately four cases of mesothelioma (Eq. 1) could potentially have been diagnosed,

$$
\text { (18 observed mesotheliomas }) *\left(\frac{1}{(1-0.17)}-1\right)
$$

\section{$=3.7$ missed mesotheliomas}

but not found on death certificates. The simulations below are based on the assumption that this expected number of missed cases actually occurred in the cohort. Based on the wide definition of potential mesothelioma codes, it was determined that there were eight ICD codes that mesothelioma could have been coded to and 83 (=95-12) deaths with these ICD codes (Table 2 ).

In each simulation, we first randomly selected 4 out of the 83 potentially misdiagnosed cases, combined them with 18 mesotheliomas, and estimated the Poisson regression coefficient as described in Section 2c (Fig. 1).

This procedure was repeated 500 times with different random draws of 4 missed mesothelioma deaths among the 83 deaths that were potentially misdiagnosed. The mean, median and $90 \%$ Bayesian confidence interval for the ratio of the joint posterior distribution of the regression coefficient based on the simulation of 22 cases to the posterior distribution of the regression coefficient based on the observed 18 cases was calculated and is presented in the top row of Table 3 .

Table 1. Pre-ICD-10 Codes which Mesothelioma Might have been Coded for Different Revisions of ICD (Years of Use in the United States are Shown for each ICD Revision)

\begin{tabular}{|c|c|c|c|c|c|}
\hline & $\begin{array}{c}\text { ICD-5 } \\
1939-48[36]\end{array}$ & $\begin{array}{c}\text { ICD-6 } \\
1949-57[37]\end{array}$ & $\begin{array}{c}\text { ICD-7 } \\
1958-67[38]\end{array}$ & $\begin{array}{c}\text { ICD-8 } \\
1968-78[39]\end{array}$ & $\begin{array}{l}\text { ICD-9 } \\
1979-98\end{array}$ \\
\hline Mesothelioma & $\begin{array}{c}46 \mathrm{~g}, 46 \mathrm{~h}, 47 \mathrm{e} \\
47 \mathrm{f}, 55 \mathrm{e}\end{array}$ & $\begin{array}{l}153,157,158,159 \\
163,164,197,199\end{array}$ & $\begin{array}{c}158,159162.2 \\
163,164,165 \\
197.9,199\end{array}$ & $\begin{array}{c}158.9,163.0,163,163.1 \\
163.9,195,195.0,195.9,199 \\
199.0,199.1\end{array}$ & $\begin{array}{c}158.8,158.9,162.9,163, \\
163.9,164.9,195.1,195.2, \\
199.0,199.1\end{array}$ \\
\hline $\begin{array}{l}\text { Mesothelioma } \\
\text { Benign codes }\end{array}$ & $56 \mathrm{e}$ & $227,229,231,239$ & $\begin{array}{c}227,229,231, \\
239\end{array}$ & $212.4,228$ & $212.4,229.9$ \\
\hline
\end{tabular}


Table 2. Pre-ICD-10 Codes/Revisions Listed in Table 1 which are Present in the Libby Worker Cohort and Mesothelioma Deaths Among these Codes

\begin{tabular}{|c|c|c|c|c|c|c|c|c|c|}
\hline ICD Code & 162.9 & 163 & 163.9 & 164 & 165 & 195.2 & 199 & 199.1 & Total \\
\hline ICD revision & $9^{\text {th }}$ & $6^{\text {th }}-9^{\text {th }}$ & $8^{\text {th }}, 9^{\text {th }}$ & $6^{\text {th }}, 7^{\text {th }}$ & $7^{\text {th }}$ & $9^{\text {th }}$ & $7^{\text {th }}, 8^{\text {th }}$ & $8^{\text {th }}, 9^{\text {th }}$ & \\
\hline Number of deaths & 64 & 2 & 4 & 1 & 1 & 2 & 1 & 20 & 95 \\
\hline Number of confirmed mesotheliomas & 2 & & 3 & & & 1 & & 6 & 12 \\
\hline
\end{tabular}

Thirteen pre-ICD-10 mesotheliomas were identified from death certificates (all coded in ICD-9) by the primary NIOSH researcher [31]. The remaining pre-ICD-10 mesotheliom case not in Table 2 was coded to asbestosis. Another five mesothelioma cases were identified from ICD-10 mesothelioma codes, resulting in total of 18 identified mesotheliomas.

The mean ratio of 1.28 implies that the estimated risk of exposure to the Libby amphibole asbestos would be approximately 1.3 times larger if all diagnosed cases of mesothelioma were recorded on the death certificates. Very similar results were obtained in alternate analysis including only possible cases with the time since first exposure of at least 30 years, to exclude deaths with short latency that are less likely to be mesotheliomas (results not shown).

\section{3b. Approach Using Specific Misdiagnoses of Mesothelioma}

The rate of misdiagnosis in the insulators cohort varied dramatically between pleural and peritoneal mesotheliomas $[17,21]$. Because information on the type of mesothelioma was only available for $1 / 3$ of the 18 observed cases, existing information was extrapolated preserving the observed ratio of 1 peritoneal to 5 pleural mesotheliomas identified on death certificates from the Libby worker cohort. This resulted in 3 peritoneal cases and 15 pleural cases. The ratio between number of pleural and peritoneal mesotheliomas varied substantially between asbestos cohorts $[40,41]$; this proportion is within the range of proportions in other cohorts. Additionally, several mesotheliomas of unspecified type in the Libby worker cohort also had a multiple cause code for asbestosis and may be more likely to be peritoneal rather than pleural [42]. Also, among mesotheliomas mentioned on the death certificate, Selikoff and Seidman [21] found that peritoneal mesothelioma was slightly more likely to be correctly identified as peritoneal, compared to the corresponding rate of correct identification for pleural mesothelioma.

Using the results of Selikoff and Seidman [21], we considered specific cancers that mesothelioma could be most likely misdiagnosed as - lung, abdominal, pancreatic and colon cancers. Using the type-specific misdiagnosis rates for pleural mesothelioma of $22.6 \%$ [21], we considered that pleural mesotheliomas were underestimated by 4 cases (Eq. 2). As lung cancer was by far the most likely misdiagnosis, these 4 additional
(15 pleural mesotheliomas $) *\left(\frac{1}{(1-0.226)}-1\right)$

\section{$=4.38$ missed pleural mesotheliomas}

cases were chosen from 111 lung cancer cases. Underestimation of peritoneal mesotheliomas was 3 additional peritoneal mesothelioma cases (Eq. 3) as peritoneal mesothelioma was identified as any mesothelioma on death certificate only in $53.7 \%$ of all confirmed peritoneal

$$
\text { (3 peritoneal mesotheliomas }) *\left(\frac{1}{(1-0.537)}-1\right)
$$

\section{$=2.59$ missed peritonealal mesotheliomas}

mesothelioma deaths [21]. The most likely [21] misdiagnosed codes for the three true peritoneal mesotheliomas would be 2 pancreatic and 1 colon cancer (abdominal cancer is the most likely misdiagnosis, but the Libby worker cohort had only 2 cases of abdominal cancers, one of which was actually identified as mesothelioma on the death certificate, and pancreatic and colon cancer were next likely misdiagnoses). The simulations below are based on the assumption that these expected numbers of missed cases actually occurred in the cohort.

In each simulation, 4 out of 111 lung cancers, 1 out of 13 colon cancers and 2 out of 12 pancreatic cancers were randomly chosen. Additionally, we used the finding of Selikoff and Seidman [21] that $7.7 \%$ of mesotheliomas on death certificates are actually not mesotheliomas. That resulted in 1 randomly chosen mesothelioma being designated as misdiagnosed in each simulation among 18 that were found on death certificates.

Therefore, in each simulation, 24 cases (17 mesotheliomas from the primary investigator's list and 7 additional mesotheliomas: 4 lung, 1 colon and 2 pancreas) were randomly chosen among cases with the corresponding diagnosis and a Poisson coefficient was estimated as described in Section 2c (Fig. 2).
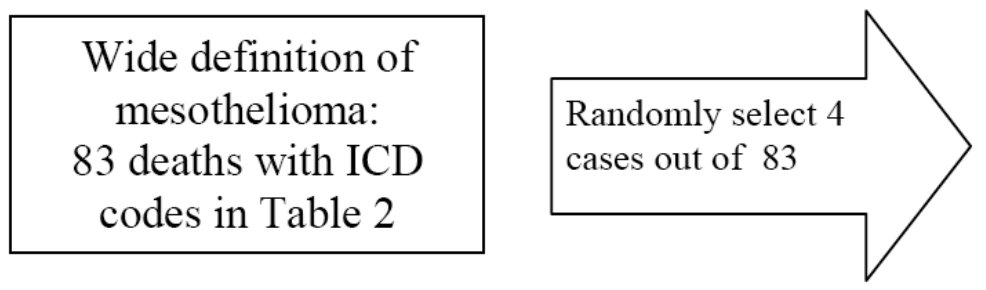
Form new dataset: 1871 workers and $22(=18+4)$ mesothelioma cases. Conduct analysis as in Section 2c and retain the distribution of $\beta$

Fig. (1). Representation of each step of the simulation process in using wide definition of mesothelioma approach. 


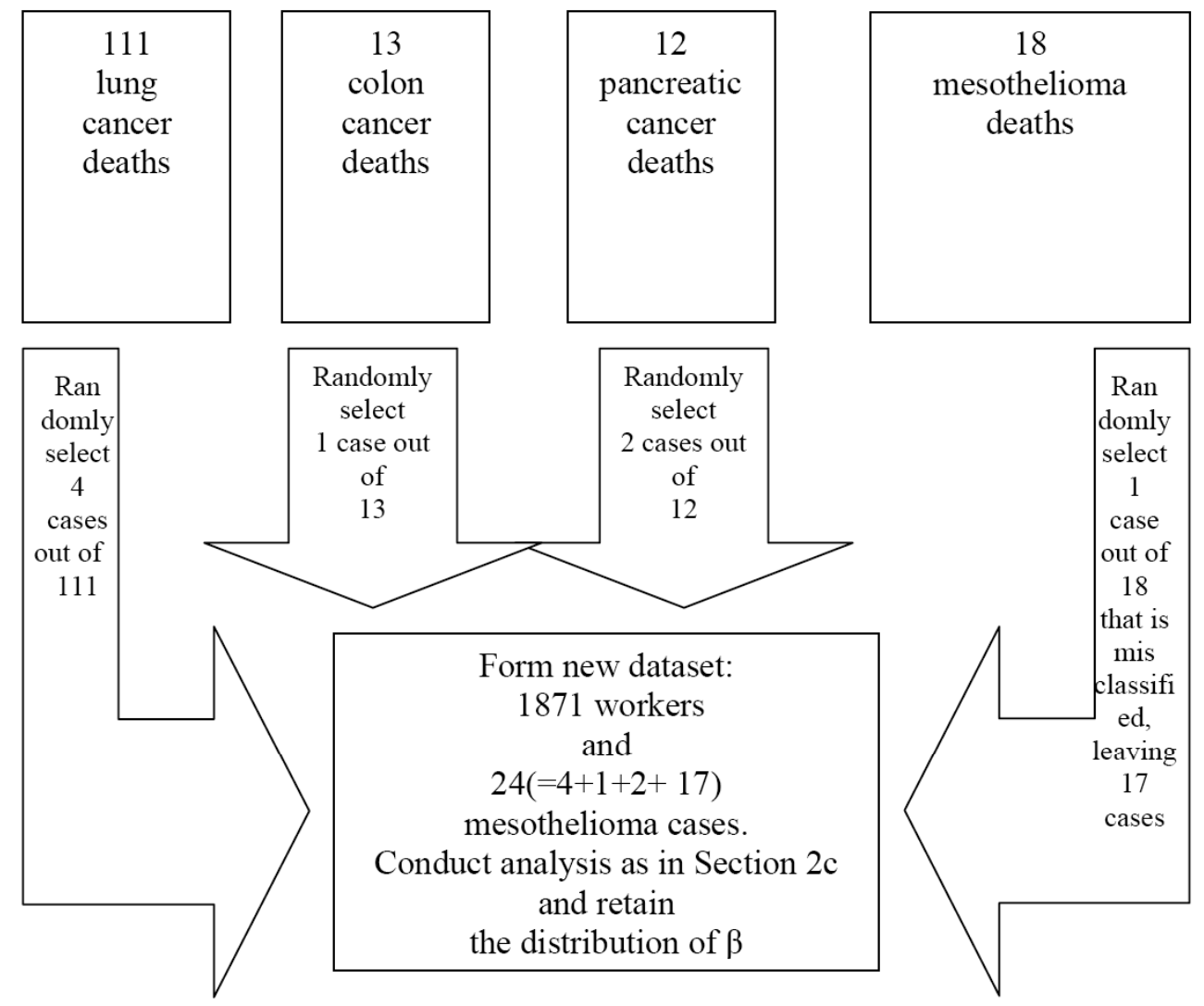

Fig. (2). Representation of the each step of the simulation process in using specific misdiagnoses of mesothelioma approach.

This procedure was repeated 500 times and the mean, median and 90\% Bayesian confidence interval for the ratio of the joint posterior distribution of the regression coefficient based on simulation of 24 cases to the posterior distribution of the regression coefficient based on the observed 18 cases are calculated and presented in the bottom row of Table 3 .

The mean ratio of 1.39 implies that the estimated risk of exposure to the Libby amphibole asbestos would be approximately 1.4 times larger if all decedents for whom medical information and pathology samples would have supported a diagnosis of mesothelioma had been identified. Very similar results were obtained in alternate analysis including only possible cases with the time since first exposure of at least 30 years, to exclude deaths with short latency that are less likely to be mesotheliomas (results not shown).
Table $\mathbf{3}$ shows that the way of defining alternative cases either wide definition of mesothelioma or specific misdiagnoses of mesothelioma, at least in this cohort, is not necessarily important; what drives underestimation of average risk in Table $\mathbf{3}$ is the number of missed cases (ratio of respective means/medians in rows of Table $\mathbf{3}$ is very close to $24 / 22$ ). That very much depends on whether information on the number of peritoneal mesotheliomas is available.

\section{DISCUSSION}

Mesothelioma underascertainment on death certificates is a persistent problem in assessing risk from asbestos that is often acknowledged, but is not generally accounted for when estimating mesothelioma risk. This paper proposes a way forward to correct downward bias in risk due to mesothelioma underascertainment.

Table 3. Monte Carlo Simulation Results for Two Approaches for Assessing the Impact of Underascertainment on Death Certificates of Mesothelioma in the Libby Worker Cohort: Distribution of the Ratio of Posteriors with Adjustment and without Adjustment

\begin{tabular}{|c|c|c|c|c|c|}
\hline \multirow{2}{*}{ Approach } & \multicolumn{4}{|c|}{$\begin{array}{l}\text { Distribution of the Ratio of Posteriors with Adjustment and without } \\
\text { Adjustment for Underascertainment of Mesothelioma }\end{array}$} & \multirow{2}{*}{$\begin{array}{l}\text { Adjusted Number of } \\
\text { Mesothelioma Cases }\end{array}$} \\
\hline & Mean & Median & \multicolumn{2}{|c|}{ Percentile } & \\
\hline Wide definition of mesothelioma & 1.28 & 1.21 & 0.73 & 2.04 & 22 cases \\
\hline
\end{tabular}


The underestimation rates of peritoneal mesothelioma are much higher than for pleural. Therefore, different approaches are appropriate depending on whether information on the proportion of peritoneal mesotheliomas among all mesotheliomas can be estimated or is known.

Results in Table $\mathbf{3}$ indicate that the method of simulation of missed cases is less important, at least for this cohort, than the estimated number of missed mesothelioma cases. Therefore, two approaches to quantifying underestimation of mesothelioma are proposed, assuming that, for pre-ICD-10 deaths, death certificates are manually inspected for mention of mesothelioma as in Davis et al. [16], possibly using only deaths with ICD codes in Table $\mathbf{1}$ for manual inspection.

If the proportion of peritoneal mesotheliomas is available, different rates of underestimation can be applied separately for pleural and peritoneal mesotheliomas. The rates are available in Selikoff and Seidman [21], based on the insulators cohort [23]: 77\% of pleural mesotheliomas and $54 \%$ of peritoneal mesotheliomas were mentioned on death certificates.

The strengths of Selikoff and Seidman [21] are threefold. First, it is one of the very few studies based on results of histopathological analysis. Second, it is by far the largest study in terms of number of subjects - it is more than 2 times as large as the next study in size [43]; and in terms of mesothelioma deaths - it includes more than 6 times more mesotheliomas than the $2^{\text {nd }}$ and $3^{\text {rd }}$ largest studies $[44,45]$ and more than 13 times more mesothelioma deaths than the $4^{\text {th }}$ largest study [46]. Also, the study represents exposure to different kinds of asbestos.

A limitation of Selikoff and Seidman [21] is that it includes only men. Peritoneal mesothelioma in women presents additional difficulty as it can be misdiagnosed as adenocarcinoma from the ovary [47], likely increasing the rate of misdiagnosis of peritoneal mesothelioma in a population.

If the proportion of peritoneal mesotheliomas is not available, the proportion of missed mesotheliomas can be derived from Davis et al. [16]. In this study of the general population, $83 \%$ of mesotheliomas recorded by the cancer registry are mentioned on death certificates. However, it is important to realize that the second approach, based solely on underestimation of mesothelioma cases on death certificates compared to incidence data based on a cancer registry, creates a lower bound for the true (but unknown) number of mesothelioma cases, as it does not account for misdiagnosed mesotheliomas that do not appear in a cancer registry. The approach following Selikoff and Seidman [21] provides a more inclusive estimate of the number of mesothelioma cases, as it includes mesotheliomas that are missed at initial diagnosis (but identifiable from existing medical records and pathology samples) as well as those that are not reported on a death certificate. However, it depends on the knowledge of the proportion of peritoneal mesotheliomas that in most cases may be only partly known. In this investigation the difference between approaches resulted in approximately 10\% difference in the estimated risk of mesothelioma.

One uncertainty in both approaches is an assumption of similarity between ICD-10 misdiagnoses and pre-ICD-10 misdiagnoses. In the Selikoff and Seidman study [21], deaths occurred in 1967-1986, more than 20 years ago, and one hopes that rates of diagnosis of mesothelioma went up with development of better medical approaches. Surprisingly, published rates of underascertainment for the early years of ICD-10 are no better than those for ICD-9 $[16,18,19]$.

On one hand, rates of misdiagnoses and miscoding of mesothelioma may decrease with continued use of ICD-10 and better diagnostic practices developed with time. On the other hand, rates of mesothelioma misdiagnoses in Selikoff and Seidman [21] are based on workers with known occupational exposure to asbestos, whereas in the general population physicians may lack a context to diagnose rare cancer like mesothelioma, especially peritoneal mesothelioma.

Another finding of Selikoff and Seidman [21] is that a small, but non-trivial proportion of cases of mesothelioma stated in death certificates are other cancers, mostly lung cancers. Our simulations accounted for that, but this finding has impact for specificity of mesothelioma diagnosis on death certificates. Therefore, published data on mesotheliomas based on death certificates has both sensitivity and specificity issues and caution is needed in interpretation and use of data on mesothelioma incidence.

The sensitivity to the assumptions in both approaches related to the misdiagnoses are low, as, at least for this cohort, the estimated number of missed mesothelioma cases dominates impact on risk estimate. However, since the main interest of this article is in average underascertainment, in the illustration of approaches in Section 3, all the misdiagnosis estimates from the published results were taken as central estimates. In reality, all the estimated rates are random variables. This variability should be accounted for in simulations, resulting in somewhat wider confidence intervals (comparison of rows of Table $\mathbf{3}$ suggests that effect on confidence bounds would not be large).

Clearly, the estimation of the proportion of peritoneal mesotheliomas needs to be as accurate as possible. It is possible, that proper diagnosis of peritoneal mesothelioma has improved in 20 years since study period of Selikoff and Seidman [21] and it will be important to modify assumptions when such data becomes available.

The cohort [21] is somewhat unusual in terms of the number of peritoneal mesotheliomas exceeding pleural (285 peritoneal vs 173 pleural), but the proportion of peritoneal mesotheliomas (153 peritoneal vs 134 pleural) based only on death certificate information (before histopathological analysis) is consistent with other asbestos cohorts. In any case, the proportion of peritoneal mesothelioma found in Selikoff and Seidman [21] is not used anywhere in the approaches of this paper.

Insufficient follow-up is another important source of underestimation of mesothelioma risk. The effect of insufficient follow-up would be to underestimate the risk of exposure since there would be workers who may eventually die of mesothelioma that are not counted at the time of the current update to the cohort. As the risk of mesothelioma is evaluated as an additive risk, the mesothelioma risk may reasonably be expected to rise as the length of follow-up increases. 
The Libby amphibole asbestos workers cohort was used for illustration and evaluation of the methodologies in this paper. It is possible that results would be somewhat different using another asbestos-exposed cohort/population, but the Libby worker cohort is a large cohort with reasonably long follow-up, so uncertainty about results is reasonably low.

In addition to use in risk assessments, another possible use of the methodology of this paper is in mesothelioma mortality predictions for other cohorts and populations. There are many efforts in this direction around the world - in Australia [48,49], Denmark [50], France [51,52], Itlay [53], the Netherlands [54], Europe-wide [55,56], Japan [57,58], the UK [59-61], and the US [62-64]. The prediction methodologies differ between efforts, but the current or past incidence is an important part of the future prediction, therefore underascertainment of current mesothelioma incidence would result in predictions biased low, as long as absolute risk models are used.

Approaches similar to those proposed here can be used for risk assessment where the outcome is a different cancer, as misclassification on death certificates is an issue for many different cancers. However, when a relative risk estimator is used, the effect of underestimation depends on whether misclassification in the death certificates of the study is the same as in the comparison population. This may or may not be true, depending on the particular cancer and study.

\section{ACKNOWLEDGEMENTS}

The authors would like to thank Paul White of EPA for helpful comments during the preparation of this manuscript.

\section{DISCLAIMER}

The findings and conclusions in this report are those of the authors and do not necessarily represent the views of the U.S. Environmental Protection Agency or the National Institute for Occupational Safety and Health. Any mention of trade names or commercial products does not constitute endorsement or recommendation for use. The authors declare that they have no competing financial interests.

\section{REFERENCES}

[1] Percy C, Dolman A. Comparison of the coding of death certificates related to cancer in seven countries. Am J Public Health 1978; 93: 335-50.

[2] Percy C, Stanek E, Gloeckler L. Accuracy of cancer death certificates and its effect on cancer mortality statistics. Am J Public Health 1981; 71: 242-50.

[3] Tomek S, Manegold C. Chemotherapy for malignant pleural mesothelioma: past results and recent developments. Lung Cancer 2004; 45: S103-19.

[4] Mossman BT, Bignon J, Corn M, Seaton A, Gee JB. Asbestos: scientific developments and implications for public policy. Science 1990; 247(4940): 294-301.

[5] Selikoff IJ, Lee DHK. Asbestos and disease. New York: Academic Press 1978.

[6] Dikensoy O. Mesothelioma due to environmental exposure to erionite in Turkey. Cur Opin Pulm Med 2008; 14 (4): 322-5.

[7] Dogan AU, Baris YI, Dogan M, et al. Genetic predisposition to fiber carcinogenesis causes a mesothelioma epidemic in Turkey. Cancer Res 2006; 66(10): 5063-8.

[8] Carbone M, Albelda SM, Broaddus VC, et al. Eighth International Mesothelioma Interest Group. Oncogene 2007; 26, 6959-67.

[9] Kroczynska B, Cutrone R, Boccheta M, et al. Crocidolite asbestos and SV40 are cocarcinogens in human mesothelial cells and in causing mesothelioma in hamsters. PNAS 2006; 103(38), 1412833 .
[10] Hofmann J, Mintzer D, Warhol M. Malignant mesothelioma following radiation therapy. Am J Med 1994; 97(4): 379-82.

[11] Andersson M, Wallin H, Jonsson M, et al. Lung carcinoma and malignant mesothelioma in patients exposed to Thorotrast: incidence, histology and p53 status. Int J Cancer 1995; 63: 330-6.

[12] Ishikawa Y, Mori T, Machinami R. Lack of apparent excess of malignant mesothelioma but increased overall malignancies of peritoneal cavity in Japanese autopsies with Thorotrast injection into blood vessels. J Cancer Res Clin Oncol 1995; 121: 567-70.

[13] Van Kaick G, Dalheimer A, Hornik S, et al. The German thorotrast study: recent results and assessment of risks. Radiat Res 1999; 152 (Suppl): S64-S71.

[14] Selikoff IJ. Use of death certificates in epidemiological studies, including occupational hazards: discordance with clinical and autopsy findings. Am J Ind Med 1992; 22(4): 469-80.

[15] Newhouse ML, Wagner JC. Validation of death certificates in asbestos workers. Br J Ind Med 1969; 26: 302-7.

[16] Davis LK, Martin TR, Kligler B. Use of death certificates for mesothelioma surveillance. Public Health Rep 1992; 107: 481-3.

[17] Ribak J, Lilis R, Suzuki Y, Penner L, Selikoff IJ. Death certificate categorization of malignant pleural and peritoneal mesothelioma in a cohort of asbestos insulation workers. J Soc Occup Med 1991; 41: 137-9.

[18] Pinheiro GA, Antao VCS, Bang KM, Attfield MD. Malignant mesothelioma surveillance: a comparison of ICD 10 mortality data with SEER incidence data in nine areas of the United States. Int J Occup Environ Health 2004; 10: 251-5.

[19] Camidge DR, Stockton DL, Bain M. Factors affecting the mesothelioma detection rate within national and international epidemiological studies: insights from Scottish linked cancer registry-mortality data. Br J Cancer 2006; 95: 649-52.

[20] WHO. International Statistical Classification of Diseases and Related Health Problems. Tenth Revision. Geneva: World Health Organization 1992.

[21] Selikoff IJ, Seidman H. Use of death certificates in epidemiological studies, including occupational hazards: variations in discordance of different asbestos-associated diseases on best evidence ascertainment. Am J Ind Med 1992; 22(4): 481-92.

[22] WHO. Manual of the International Statistical Classification of Diseases, Injuries, and Causes of Death. Ninth Revision. Geneva: World Health Organization 1977.

[23] Selikoff IJ, Churg J, Hammond EC. Asbestos exposure and neoplasia. JAMA 1964; 188: 22-6.

[24] McDonald JC, McDonald AD, Armstrong B, Sebastien P. Cohort study of mortality of vermiculite miners exposed to tremolite. Br J Ind Med 1986; 43: 436-44.

[25] McDonald JC, Sebastien P, Armstrong B. Radiological survey of past and present vermiculite miners exposed to tremolite. $\mathrm{Br} \mathrm{J}$ Ind Med 1986; 43: 445-9.

[26] Amandus HE, Wheeler R, Jankovic J, Tucker J. The morbidity and mortality of vermiculite miners and millers exposed to tremoliteactinolite: part I. Exposure estimates. Am J Ind Med 1987; 11: 114.

[27] Amandus HE, Wheeler R. The morbidity and mortality of vermiculite miners and millers exposed to tremolite-actinolite: Part II. Mortality. Am J Ind Med 1987; 11: 15-26.

[28] Amandus HE, Althouse R, Morgan WK, Sargent EN, Jones R. The morbidity and mortality of vermiculite miners and millers exposed to tremolite-actinolite: Part III. Radiographic findings. Am J Ind Med 1987; 11: 27-37.

[29] Amandus HE, Wheeler R, Armstrong BG, McDonald AD McDonald JC, Sebastien P. Mortality of vermiculite miners exposed to tremolite. Ann Occup Hyg 1988; 32: 459-67.

[30] McDonald JC, Harris J, Armstrong B. Mortality in a cohort of vermiculite miners exposed to fibrous amphibole in Libby, Montana. Occup Environ Med 2004; 61: 363-6.

[31] Sullivan PA. Vermiculite, respiratory disease, and asbestos exposure in Libby, Montana: update of a cohort mortality study. Environ Health Perspect 2007; 115: 579-85.

[32] Whitehouse AC. Asbestos-related pleural disease due to tremolite associated with progressive loss of lung function: serial observations in 123 miners, family members, and residents of Libby, Montana. Am J Ind Med 2004; 46(3): 219-25.

[33] Marsh GM, Gula MJ, Youk AO, Buchanich JM, Churg A, Colby TV. Historical cohort study of US man-made vitreous fiber 
production workers: II. Mortality from mesothelioma. J Occup Environ Med 2001; 43: 757-66.

[34] Hillerdal G. Malignant mesothelioma: review of 4710 published cases. Br J Dis Chest 1983; 77: 321-43.

[35] Spiegelhalter D, Thomas A, Best N. WinBugs Version 1.4 User Manual. 2003; Available at: http://www.mrcbsu.cam.ac.uk/bugs/winbugs/manual14.pdf.

[36] WHO. Manual of the International Statistical Classification of Diseases, Injuries, and Causes of Death. Fifth Revision. Geneva: World Health Organization 1938

[37] WHO. Manual of the International Statistical Classification of Diseases, Injuries, and Causes of Death. Sixth Revision. Geneva: World Health Organization 1948.

[38] WHO. Manual of the International Statistical Classification of Diseases, Injuries, and Causes of Death. Seventh Revision. Geneva: World Health Organization 1957.

[39] WHO. Manual of the International Statistical Classification of Diseases, Injuries, and Causes of Death. Eighth Revision. Geneva: World Health Organization 1967.

[40] Hodgson JT, Darnton A. The quantitative risks of mesothelioma and lung cancer in relation to asbestos exposure. Ann Occup Hyg 2000; 44(8): 565-601.

[41] Boffetta P. Epidemiology of peritoneal mesothelioma: a review. Ann Oncol 2007; 18: 985-90.

[42] Roggli VL, Vollmer RT. Twenty-five years of fiber analysis: what have we learned? Hum Pathol 2008; 39: 307-15.

[43] Sluis-Cremer GK, Liddell FD, Logan WP, Bezuidenhout BN. The mortality of amphibole miners in South Africa, 1946-80. Br J Ind Med 1992; 49: 566-75.

[44] Magnani C, Terracini B, Ivaldi C, Mancini A, Botta M. Tumor mortality and from other causes in asbestos cement workers at the Casale Montferrato plant. Med Lav 1996; 87:133-46.

[45] de Klerk NH, Musk AW, Armstrong BK, Hobbs MST. Diseases in miners and millers of crocidolite from Wittenoom, Western Australia: a further follow-up to December 1986. Ann Occup Hyg 1994; 38(Supp1), 647-55.

[46] Liddell FDK, McDonald AD, McDonald JC. The 1891-1920 cohort of Quebec chrysotile miners and millers: development from 1904 and mortality to 1992. Ann Occup Hyg 1997; 41, 13-36.

[47] Krasuski P, Poniecka A, Gal E. The diagnostic challenge of peritoneal mesothelioma. Arch Gynecol Obstet 2002; 266: 130-2.

[48] Berry G. Prediction of mesothelioma, lung cancer, and asbestosis in former Wittenoom asbestos workers. Br J Ind Med 1991; 48: 793802 .

[49] Clements M, Berry G, Shi J, Ware S, Yates D, Johnson A. Projected mesothelioma incidence in men in New South Wales. Occup Environ Med 2007; 64(11): 747-52.
[50] Kjaergaard $\mathrm{J}$, Andersson $\mathrm{M}$. Incidence rates of malignant mesothelioma in Denmark and predicted future number of cases among men. Scand J Work Environ Health 2000; 26(2): 112-7.

[51] Ilg AG, Bignon J, Valleron AJ. Estimation of the past and future burden of mortality from mesothelioma in France. Occup Environ Med 1998; 55(11): 760-5.

[52] Banaei A, Auvert B, Goldberg M, Gueguen A, Luce D, Goldberg S. Future trends in mortality of French men from mesothelioma. Occup Environ Med 2000; 57(7): 488-94.

[53] Gasparrini A, Pizzo AM, Gorini G, et al. Prediction of mesothelioma and lung cancer in a cohort of asbestos exposed workers. Eur J Epidemiol 2008; 23(8): 541-6.

[54] Segura O, Burdorf A, Looman C. Update of predictions of mortality from pleural mesothelioma in the Netherlands. Occup Environ Med 2003; 60(1): 50-5.

[55] Peto J, Decarli A, La Vecchia C, Levi F, Negri E. The European mesothelioma epidemic. Br J Cancer 1999; 79(3-4): 666-72.

[56] Pelucchi C, Malvezzi M, La Vecchia C, Levi F, Decarli A, Negri E. The Mesothelioma epidemic in Western Europe: an update. Br J Cancer 2004; 90(5): 1022-4.

[57] Murayama T, Takahashi K, Natori Y, Kurumatani N. Estimation of future mortality from pleural malignant mesothelioma in Japan based on an age-cohort model. Am J Ind Med 2006; 49(1): 1-7.

[58] Azuma K, Uchiyama I, Chiba Y, Okumura J. Mesothelioma risk and environmental exposure to asbestos: past and future trends in Japan. Int J Occup Environ Health 2009; 15: 166-72.

[59] Peto J, Hodgson JT, Matthews FE, Jones R. Continuing increase in mesothelioma mortality in Britain. Lancet 1995; 345(8949): 535-9.

[60] Hodgson JT, Peto J, Jones R, Matthews FE. Mesothelioma mortality in Britain: patterns by birth cohort and occupation. Ann Occup Hyg 1997; 41(Suppl 1): 129-33.

[61] Hodgson JT, McElvenny DM, Darnton AJ, Price JM, Peto J. The expected burden of mesothelioma mortality in Great Britain from 2002 to 2050. Br J Cancer 2005; 92(3): 587-93.

[62] Nicholson WJ, Perkel G, Selikoff IJ. Occupational exposure to asbestos: population at risk and projected mortality - 1980-2030. Am J Indust Med 1982; 3: 259-311.

[63] Price B, Ware A. Time trend of mesothelioma incidence in the United States and projection of future cases: An update based on SEER data for 1973 through 2005. Crit Rev Toxicol 2009; 39(7): 576-88.

[64] Moolgavkar SH, Meza R, Turim J. Pleural and peritoneal mesotheliomas in SEER: age effects and temporal trends, 19732005. Cancer Causes Control 2009; 20(6): 935-44.

(C) Kopylev et al.; Licensee Bentham Open.

This is an open access article licensed under the terms of the Creative Commons Attribution Non-Commercial License (http://creativecommons.org/licenses/by$\mathrm{nc} / 3.0 /$ ) which permits unrestricted, non-commercial use, distribution and reproduction in any medium, provided the work is properly cited. 